\title{
HIV versus the immune system: another apparent victory for the virus
}

\author{
Norman L. Letvin ${ }^{1}$ and Bruce D. Walker ${ }^{2}$ \\ ${ }^{1}$ Division of Viral Pathogenesis, Beth Israel Deaconess Medical Center, and \\ ${ }^{2}$ Partners AIDS Research Center, Massachusetts General Hospital, Harvard Medical School, Boston, Massachusetts, USA \\ Address correspondence to: Bruce D. Walker, Partner AIDS Research Center, Massachusetts General Hospital, \\ Harvard Medical School, 149 13th Street, Room 5212 D, Boston, Massachusetts 02129, USA. \\ Phone: (617) 724-8332; Fax: (617) 726-4691; E-mail: bwalker@helix.mgh.harvard.edu.
}

The only realistic hope for curbing the expanding HIV pandemic is the development of an effective vaccine. Understanding the immune responses that might protect from infection is of utmost relevance to this effort. With the most recent global figures showing that over 36 million people are now living with HIV, and expectations for rapidly expanding epidemics in Asia and the former Soviet Union, the need to understand immune protection in HIV infection is paramount.

One group that has generated intense interest regarding potential insights into protective immunity consists of people with repeated exposures to HIV that remain seronegative. In particular, early studies showed some commercial sex workers in Kenya remained persistently seronegative, despite daily exposure to numerous HIV-infected sex partners (1). Approximately $10 \%$ of individuals in this cohort were thus termed "resistant" to HIV infection, and many of these resistant individuals were found to have detectable, albeit lowlevel, cytotoxic T lymphocyte (CTL) responses in their blood (2). Since CTLs can at least theoretically kill virus-infected cells before progeny viruses are produced, it was hypothesized that CTLs were actually mediating the protection. In this issue of the JCI, Kaul et al. now provide much-anticipated follow-up on this cohort, showing that the resistance is indeed relative at best, as a significant number of these individuals have gone on to seroconvert (3). However, important clues contained in the manuscript by Kaul et al. may help to dissect further the immune responses that will be required for long-lasting protection.

In the present study, Kaul et al. (3) have documented late seroconversion to HIV in approximately $10 \%$ (11 of 114) of sex workers who were initially deemed resistant to HIV infection. In a

case control analysis, they report that seroconversion was associated with a reduction in sex work, defined as either a decrease in daily clients of two or more, or a break from commercial sex work for over 2 months. The actual percentages with reduction in sex work were $91 \%$ for seroconverters versus $45 \%$ for those remaining seronegative, rendering a $P$ value of 0.03 . Certainly in part due to the difficulties in conducting research in developing countries, only six of the eleven late seroconverters were monitored for CTLs. Four had documented CTLs prior to seroconverting, albeit at very low levels. The presence of CTLs and the reduced sex work led to an expanded hypothesis that perhaps waning immunity was responsible for the late susceptibility to infection. This hypothesis prompted longitudinal studies that are interpreted to show that reduction in sex work is associated with declines in CTLs. However, missing from the longitudinal studies thus far is evidence that individuals with reductions in sex work and loss of CTLs actually have a higher incidence of new HIV infection.

\section{Approximately $10 \%$ of individuals in this cohort were termed "resistant" to HIV infection, and many of these resistant individuals were found to have detectable, albeit low-level, cytotoxic T lymphocyte responses in their blood.}

The hypothesis raised by Kaul et al. (3) that CTLs are actually protective in this cohort is based on irrefutable evidence that CTLs are a protective host defense in persons in whom HIV infection is established. The most compelling evidence comes from studies of an AIDS virus infection in monkeys, in which CD8 cell-depletion (and thus CTL depletion) in vivo leads to dramat- ic increases in steady-state viremia, which subsequently drop again as CTLs return $(4,5)$. Others have shown a negative correlation between CTLs and viral load in untreated individuals, with those having the highest CTL levels having the lowest viral loads (6). Additional studies show that the drop in high-level viremia seen in acute infection coincides with the development of CTLs (7-9). The antiviral effect is mediated by CTL recognition of processed viral proteins on the surface of the infected cell, which serves as a signal that the cell contains foreign material and must be eliminated. Not only do CTLs lyse infected cells, they also produce antiviral factors that serve to inhibit progeny virions that may have already been produced $(10,11)$. In some people with stable nonprogressing HIV infection, persistent levels of HIVdirected CTLs in excess of $10 \%$ of CD8 cells may be observed in the setting of a persistently undetectable viral load (B.D. Walker, unpublished data).

The notion that reduction in sex work and resultant loss of CTLs may actually be a risk factor for HIV infection in this 
occurred because of HIV infection is dismissed by the authors based on responses of the sex workers to questionnaires, but closer monitoring of the individuals and their sexual contacts through the period of seroconversion should answer this more definitively. The differences between CTL levels in HIV-infected individuals and those observed in the persistently seronegative sex workers are quite striking for the very modest levels in the latter group. Other methods of analysis such as the use of methods to visualize CTLs directly by flow cytometry (12) will help to solidify the argument that CTLs are indeed present, and may help to determine whether subsequent seroconversion is related to a defect in function of CTLs (13, 14). Other immune parameters, such as mucosal immune responses, will be important to monitor with a similar degree of detail. The data already indicate that there must be multifactorial issues related to late seroconversion, since two of the eleven sex workers seroconverted without a documented break in sex work.

The mechanism that might be responsible for the elicitation of HIV-specific $\mathrm{CD}^{+}$CTLs in repeatedly HIV-exposed, seronegative individuals remains to be defined. Under usual circumstances, CTLs that recognize viral proteins are generated only when viral protein synthesis occurs in a cell and peptide fragments of those proteins are generated that can bind to $\mathrm{MHC}$ class I proteins for presentation to $\mathrm{CD}^{+}$lymphocytes. Since these virus-exposed individuals do not harbor sufficient replicating HIV systemically to seroconvert, this mechanism is unlikely to explain their generation of CTLs. Kaul et al. (3) have suggested that episodes of self-limited, anatomically contained HIV replication might account for the persistent boosting of CTL responses in these seronegative individuals. The fact that no temporally or anatomically contained HIV replication in humans has been documented argues against this explanation. There is, however, compelling evidence that exposure to one virus can prime the immune system for CTLs that recognize another virus $(15,16)$. Additionally, exposure to apoptotic infected cells that are not infectious can lead to induction of CTL responses (17), a phenomenon termed cross-priming, which might explain the detection of HIV-specific CTLs in the absence of infection in the subjects evaluated in this study.
Whether priming of CTLs has occurred in these individuals as a result of exposure to HIV or to another pathogen, this study suggests that immunity to HIV wanes as the commercial sex workers take a holiday from their usual working routine. Loss of CTLs would presumably occur because of the elimination of antigenic exposure to these individuals. Implicit in this suggestion is the assumption that persistent exposure to antigen is required for persistent immunologic memory. In fact, that assumption remains a controversial issue among immunologists. Certainly, humoral immunity to a protein antigen declines in the years following immunization with vaccine antigens such as tetanus toxoid or inactivated poliovirus. However, studies in murine systems also have indicated that memory $\mathrm{CD}^{+}{ }^{+} \mathrm{T}-\mathrm{lym}$ phocyte populations can persist and even divide over time in the absence of antigen (18). If the observations of Kaul et al. (3) are indeed correct, these findings provide evidence that $\mathrm{CD}^{+}$T-lymphocyte immunity can decay rapidly in the absence of antigen. In fact, the rapidity of decline would be greater than that observed in natural HIV infection when persons are started on highly active antiretroviral therapy (HAART), and viremia is reduced to undetectable levels $(19,20)$.

Finally, the authors of this study (3) explain the persistent HIV seronegativity of this small subset of commercial sex workers by suggesting that virus-specific CTLs can confer or contribute to frank protection against HIV infection. The very nature of how CTLs mediate antiviral activity does not suggest that they should necessarily be able to provide absolute protection against infection by a virus. $\mathrm{CD}^{+} \mathrm{T}$ lymphocytes can only recognize viral antigen expressed on the surface of an infected cell. Thus, CTLs should be able to contain the spread of an established viral infection but not necessarily block the establishment of such an infection. Recent AIDS vaccine studies in nonhuman primate AIDS models, in fact, show that high frequency CTL responses elicited by vaccination can control viral loads but not prevent infection following virus challenge $(21,22)$. It remains possible, however, that $\mathrm{CD}^{+} \mathrm{T}$-lymphocyte functions other than cytolytic activity may contribute to antiviral activity in the Kenyan commercial sex workers. These functions might include the release of $\beta$ chemokines and/or a variety of cytokines $(10,11)$. Alternatively, other immune responses not measured in the present study, particularly those that might operate at the mucosal level (23), may contribute to protection.

Importantly, this study by Kaul et al. (3) leaves a number of issues still to be resolved. Recent AIDS vaccine studies in nonhuman primates have indicated that pre-existing virus-specific CTL responses can confer substantial clinical protection following infection, with lower virus loads, preserved $\mathrm{CD}^{+}$ T-lymphocyte numbers and delays in the onset of clinical AIDS $(21,22)$. Moreover, Rosenberg et al. have shown that HIV replication can be controlled without therapy in a group of HIVinfected individuals in whom antiviral therapy was transiently instituted before a significant loss of $\mathrm{CD}^{+} \mathrm{T}$ lymphocytes had occurred (24). It will be very important to monitor the clinical course of AIDS in these HIVinfected commercial sex workers. This unusual subpopulation of individuals, because of pre-existing memory CTLs specific for HIV prior to their infections, may actually have relatively preserved $\mathrm{CD}^{+} \mathrm{T}$-lymphocyte populations and therefore sufficient immune function to control their HIV infections effectively in the absence of antiviral drug therapy. The individuals described by Kaul et al. may therefore have relatively benign clinical disease courses in the coming years. Continued scrutiny of unique cohorts such as these will likely provide additional insights that will aid in the quest for an effective HIV vaccine.

1. Fowke, K.R., et al. 1996. Resistance to HIV-1 infection among persistently seronegative prostitutes in Nairobi, Kenya. Lancet. 348:1347-1351.

2. Rowland-Jones, S.L., et al. 1998. Cytotoxic T cell responses to multiple conserved HIV epitopes in HIV-resistant prostitutes in Nairobi. J. Clin. Invest. 102:1758-1765.

3. Kaul, R., et al. 2001. Late seroconversion in HIVresistant Nairobi prostitutes despite pre-existing $\mathrm{HIV}$-specific $\mathrm{CD}^{+}$responses. J. Clin. Invest. 107:341-349.

4. Schmitz, J.E., et al. 1999. Control of viremia in simian immunodeficiency virus infection by CD8+ lymphocytes. Science. 283:857-860.

5. Jin, X., et al. 1999. Dramatic rise in plasma viremia after CD8(+) T cell depletion in simian immunodeficiency virus-infected macaques. J. Exp. Med. 189:991-998.

6. Ogg, G.S., et al. 1998. Quantitation of HIV-1-specific cytotoxic T lymphocytes and plasma load of viral RNA. Science. 279:2103-2106.

7. Koup, R.A., et al. 1994. Temporal association of cellular immune responses with the initial control of viremia in primary human immunodeficiency virus type 1 syndrome. J. Virol. 68:4650-4655. 
8. Borrow, P., Lewicki, H., Hahn, B.H., Shaw, G.M., and Oldstone, M.B.A. 1994. Virus-specific CD8+ cytotoxic T-lymphocyte activity associated with control of viremia in primary human immunodeficiency virus type 1 infection. J. Virol. 68:6103-6110.

9. Kuroda, M.J., et al. 1999. Emergence of CTL coincides with clearance of virus during primary simian immunodeficiency virus infection in rhesus monkeys. J. Immunol. 162:5127-5133.

10. Yang, O.O., et al. 1997. Suppression of human immunodeficiency virus type 1 replication by CD8+ cells: evidence for HLA class I-restricted triggering of cytolytic and noncytolytic mechanisms. J. Virol. 71:3120-3128.

11. Wagner, L., et al. 1998. Beta-chemokines are released from HIV-1-specific cytolytic T-cell granules complexed to proteoglycans. Nature. 391:908-911.

12. Altman, J.D., et al. 1996. Phenotypic analysis of antigen-specific $\mathrm{T}$ lymphocytes. Science. 274:94-96.

13. Zajac, A.J., et al. 1998. Viral immune evasion due to persistence of activated T cells without effector function. J. Exp. Med. 188:2205-2213.

14. Appay, V., et al. 2000. HIV-specific CD8(+) T cells produce antiviral cytokines but are impaired in cytolytic function. J. Exp. Med. 192:63-75.

15. Selin, L.K., Nahill, S.R., and Welsh, R.M. 1994 Cross-reactivities in memory cytotoxic $\mathrm{T}$ lymphocyte recognition of heterologous viruses. J. Exp. Med. 179:1933-1943.

16. Selin, L.K., Varga, S.M., Wong, I.C., and Welsh, R.M. 1998. Protective heterologous antiviral immunity and enhanced immunopathogenesis mediated by memory $\mathrm{T}$ cell populations. J. Exp. Med. 188:1705-1715.

17. Albert, M.L., Sauter, B., and Bhardwaj, N. 1998 Dendritic cells acquire antigen from apoptotic cells and induce class I-restricted CTLs. Nature. 392:86-89.

18. Lau, L.L., Jamieson, B.D., Somasundaram, T., and Ahmed, R. 1994. Cytotoxic T-cell memory without antigen. Nature. 369:648-652.

19. Ogg, G.S., et al. 1999. Decay kinetics of human immunodeficiency virus-specific effector cytotox- ic T lymphocytes after combination antiretroviral therapy. J. Virol. 73:797-800.

20. Kalams, S.A., et al. 1999. Levels of human immunodeficiency virus type 1 -specific cytotoxic T-lymphocyte effector and memory responses decline after suppression of viremia with highly active antiretroviral therapy. J. Virol. 73:6721-6728.

21. Barouch, D.H., et al. 2000. Augmentation of immune responses to HIV-1 and simian immunodeficiency virus DNA vaccines by IL- $2 / \mathrm{Ig}$ plasmid administration in rhesus monkeys. Proc. Natl. Acad. Sci. USA. 97:4192-4197.

22. Barouch, D.H., et al. 2000. Control of viremia and prevention of clinical AIDS in rhesus monkeys by cytokine-augmented DNA vaccination. Science. 290:486-492.

23. Kaul, R., et al. 2000. HIV-1-specific mucosal CD8+ lymphocyte responses in the cervix of HIV-1resistant prostitutes in Nairobi. J. Immunol. 164:1602-1611.

24. Rosenberg, E.S., et al. 2000. Immune control of HIV-1 after early treatment of acute infection. Nature. 407:523-526. 